\title{
Nitric Oxide Synthase Expression in Naturally Infected Sheep Brain with Listeria monocytogenes and Relationship with Cell Death
}

\author{
Mehmet Önder Karayiğit
}

\begin{abstract}
Background: Nitric oxide is synthesized from L-arginine and catalyzed by a family of NOS. There are three different NOS isoforms: neuronal (nNOS), inducible (iNOS) and endothelial (eNOS). Nitric oxide is an important apoptosis regulator in mammalian system that can induce and prevent apoptosis depending on levels of NO production and environmental conditions of the cell. NOS expression and its relationship with apoptosis has not been well elucidated in listerial meningoencephalitis in sheep. The aim of this study was to investigate eNOS and iNOS expressions in the brain of sheep with natural listeriosis and to compare them with apoptosis which is shaped in the region.

Materials, Methods \& Results: In the study, formalin fixed and paraffin embedded brainstem tissue from 25 sheep naturally infected with LM were used from archives. Five $\mu \mathrm{m}$-thick section was taken from each block. Histopathologically, sections were stained with H\&E. Five normal sheep brain tissues were used as control. At the end of the study, Histopathologically in brainstem tissue infected with LM, multifocal microabscesses in different sizes mixed with neutrophils and macrophages were detected and perivascular mononuclear cell infiltration and meningitis characterized by mononuclear cell infiltration were found. All sections were also immunohistochemically stained with LM, eNOS and iNOS antibodies. In addition, TUNEL method was used to determine apoptosis in brain tissues. As a result of immunostaining, listeria immunoreactivity was observed in microabscesses. The Listeria antigens were detected mainly in the cytoplasm of the neutrophils and macrophages and located extracellulary in microabscesses. Both eNOS and iNOS immunoreactivity were observed in very few neurons and glial cells in normal control sheep. Neurons and glial cells in brain tissues of infected animals stained with eNOS and iNOS. But, eNOS and iNOS expressions in listeriosis animals more higher than in control and the this difference was statistically significant $(P<0.05)$. At the same time, TUNEL immunopositivity was observed mainly in the nuclei of neuron and glial cells and this findings was found to be significantly increased compared to the control group $(P<0.05)$. Discussion: The pathogenesis of listerial meningoencephalitis depends on many factors and is still a subject of research. NO is a pluripotent regulator of diverse cellular functions. NOS can trigger cellular damage. However, there are studies suggesting that it can prevent oxidative stress. eNOS and iNOS expressions may vary depending on disease and animal species. Increased levels of NO have been reported to induce apoptosis in many diseases. However, there are studies indicating that NO is able to prevent apoptosis according to the isoform in which it is synthesized. eNOS and iNOS have not been reported together expressions and with their relationship apoptosis in sheep brain with listerial meningoencephalitis. This is the first study of eNOS and iNOS expression and and its relation to cell death in sheep brain infected with LM. Our findings are consistent with previous studies suggesting that NO expression is effective on the pathogenesis of some disease in the central nervous system. However, there are studies that do not match the findings of the present study. This suggests that the role of NO synthesis in the pathogenesis of diseases may vary according to the amount of expression, type of disease and tissue, or animal species. In the present study showed that the expression of both eNOS and iNOS with increased TUNEL positive cells was statistically significant in the listeriosis compared the control brain tissue. These results suggests that eNOS and iNOS can be expressed by diverse brain cells in the pathogenesis of listeriosis in sheep. In addition this, synthesis of NO can induce the apoptosis in sheep brain with listerial meningoencephalitis.
\end{abstract}

Keywords: listeriosis, brain, eNOS, iNOS, apoptosis.

DOI: $10.22456 / 1679-9216.88907$ 


\section{INTRODUCTION}

Listeria monocytogenes (LM) is a facultative intracellular pathogen and can cause serious diseases. In animals, listeriosis usually shows a wide variety of clinical syndromes such as septicemia, abortion, meningoencephalitis, purulent conjunctivitis and mastitis $[13,28]$. In the histopathological diagnosis of encephalitic listeriosis, extensive mononuclear perivascular cell infiltration and microabscesses formation are important findings, especially in the brainstem [13,28]. Lesions consist of neutrophils, macrophages and lymphocytes. This indicates that inflammation cells play an important role in brain damage $[6,9]$.

Nitric oxide (NO) is synthesized from L arginine by nitric oxide synthase (NOS). There are two forms of the enzyme: Constructive NOS and inducible (iNOS). Constituve NOS consists of neuronal nNOS and endothelial eNOS $[16,27]$. NOS in the central nervous system is expressed either constitutively or when stimulated by a suitable stimulus such as autoimmune encephalomyelitis [8]. NO has the ability to regulate the survival and death of cells. It can close or open apoptitotic pathways depending on NO concentration and exposure time $[7,10]$.

To the best of our knowledge, eNOS and iNOS expressions and relationship with apoptosis in sheep brain with listerial meningoencephalitis has not been reported. Therefore, the aim of this study was to investigate eNOS and iNOS expressions in sheep brain with listeriosis and to compare them with apoptosis which is shaped in the region.

\section{MATERIALS \& METHODS}

\section{Animals}

In this study, 25 paraffin embedded brain tissue blocks taken from the pons and medulla oblongata regions of naturally infected with LM in sheep were used. Five sheep brain tissue blocks that died due to other diseases which not affected of central nervous system was used as control. Five $\mu$ m-thick sections were taken from brainstem and deparaffinized in xylene and then rehydrated in graded alcohols and stained with hematoxylin eosin (H\&E) by routine histopathologic tecniques. Immunohistochemically (IHC) all infected brain and control tissues were stained with eNOS (Anti eNOS polyclonal antibody) 1 , iNOS (Anti iNOS polyclonal antibody) ${ }^{1}$ and Listeria (Anti Listeria polyclonal antibody) ${ }^{2}$ antibodies. Sections blocked and incubated with primary antibodies for $1 \mathrm{~h}$. After washes two times biotinylated seconder antibody and avidinbiotin peroxidase complex (thermo) ${ }^{1}$ were applied respectively for $20 \mathrm{~min}$. Staining was done 3-amino9-ethylcarbazole (AEC) and then were counterstained with hematoxylin. All infected and control tissues from brainstem were stained by TUNEL method (In Situ Cell Death Detection Kit) ${ }^{3}$.

\section{Cell counting method}

Cells labeled positive with eNOS, iNOS and TUNEL were scored semiquantitatively. Cells showing positive reactivity were evaluated at x 20 magnification using an eyepiece with grids of 100 squares from 10 adjacent areas in equal area for each slide. The percentages of labeled cells in the region were scored as follows: (0) no staining, (1) area of staining cells < $10 \%,(2)$ area of staining cells $=10 \%-30 \%$, , (3) area of staining cells $>30 \%$.

\section{Statistical analysis}

The Mann-Whitney U Test was performed on SPSS 15.0 program in order to test whether there was a significant difference between the severity of the disease in experimental and control group animals. A significant difference was found between the experimental and control groups for eNOS, iNOS and TUNEL staining (Table 1)

Table 1. The difference between group averages is statistically significant.

\begin{tabular}{cccc}
\hline & $\begin{array}{c}\text { Experimental } \\
\text { group }\end{array}$ & Control group & $P<0.05$ \\
eNOS & $1.37 \pm 0.765$ & $0.40 \pm 0.548$ & $0.003^{*}$ \\
iNOS & $1.67 \pm 0.884$ & $0.80 \pm 0.837$ & $0.025^{*}$ \\
& & & \\
TUNEL & $1.63 \pm 0.718$ & $0.80 \pm 0.447$ & $0.005^{*}$ \\
\hline
\end{tabular}

*Significant.

\section{RESULTS}

The lesions in the brainstem of infected sheep consisted of meningitis and perivascular cell infiltration including lymphocytes, macrophages. Characteristically microabscesses foci composed of neutrophils were observed in the area (Figure 1). Listeria immunoreactivity was observed in microabscesses (Figure 1) 
M.Ö. Karayiğit. 2018. Nitric Oxide Synthase Expression in Naturally Infected Sheep Brain with Listeria monocytogenes and Relationship with Cell Death.

Acta Scientiae Veterinariae. 46: 1617.

and macrophages in perivascular cuff (Figure 2). The listeria antigens were detected mainly in the cytoplasm of the neutrophils and macrophages and located extracellulary in microabscesses (Figure 1).

Immunohistochemical staining with eNOS revealed positive reaction in glial cells (Figure 2), neurons and endothelial cells (Figure 3). In iNOS staining, a reaction was detected in neurons, glial cells, some macrophages in brainstem (Figure 3). eNOS and iNOS expressions in neurons and glial cells of infected sheep were higher than control animals and this difference was statistically significant $(P<0.05)$. TUNEL reaction occurred in the nucleus of the neurons and glial cell. In comparison with controls, the number of TUNEL-positive apoptotic neurons and glial cells in animals infected with LM were higher than control brains (Figure 3) $[P<0.05]$. There was also a positive correlation between eNOS and iNOS expressions and the number of TUNEL positive cells.

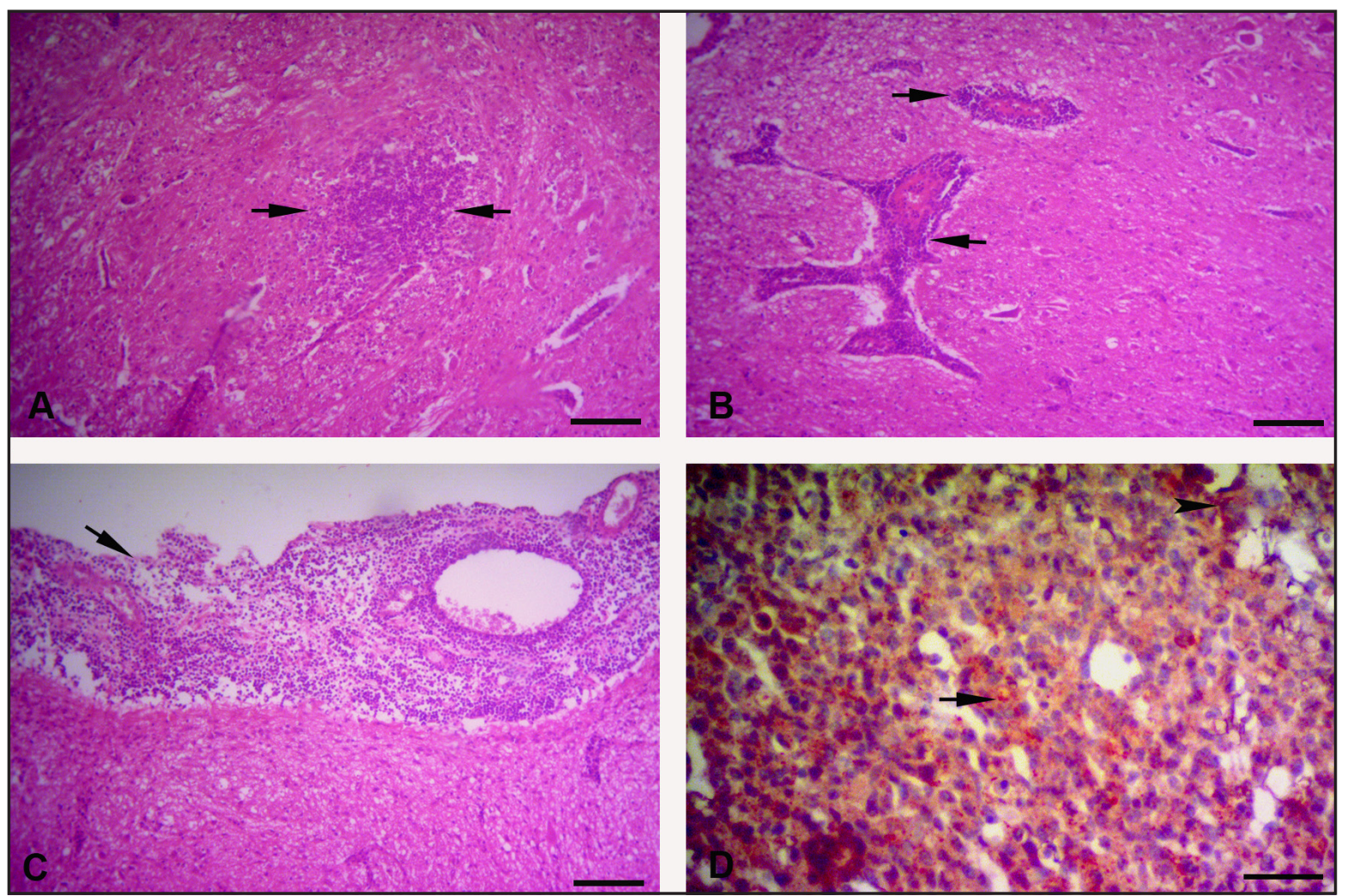

Figure 1. A- Microabscess foci (arrows). H\&E [Bar= $100 \mu \mathrm{m}]$. B- Perivascular cuff (arrows). H\&E [Bar= $100 \mu \mathrm{m}]$. C- Meningitis (arrow). $\mathrm{H} \& \mathrm{E}[\mathrm{Bar}=100 \mu \mathrm{m}]$. D- Immunolabelling macrophages with listeria antigens (arrow head) and extracellular antigens in microabscess (arrow). IHC $[\mathrm{Bar}=25 \mu \mathrm{m}]$.

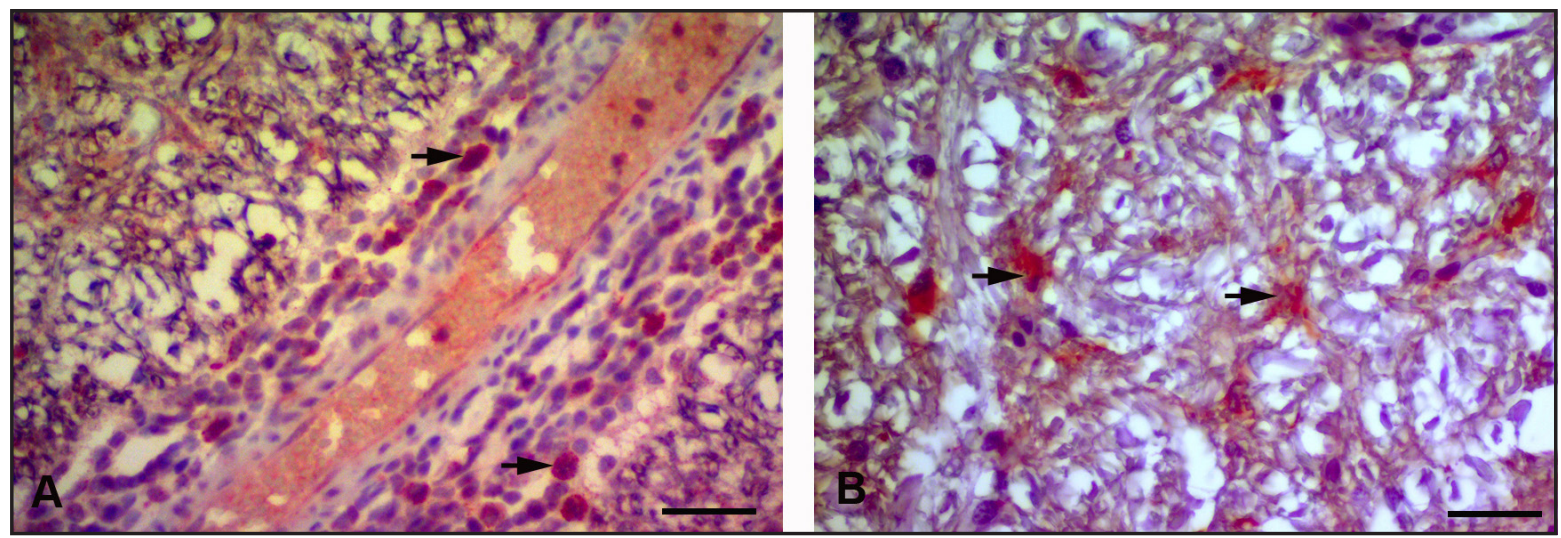

Figure 2. A- Immunolabelling macrophages with listeria antigens (in perivascular cuff (arrows). IHC [Bar= $25 \mu \mathrm{m}]$. B- eNOS positive glial cells (arrows). IHC [Bar= $25 \mu \mathrm{m}]$. 


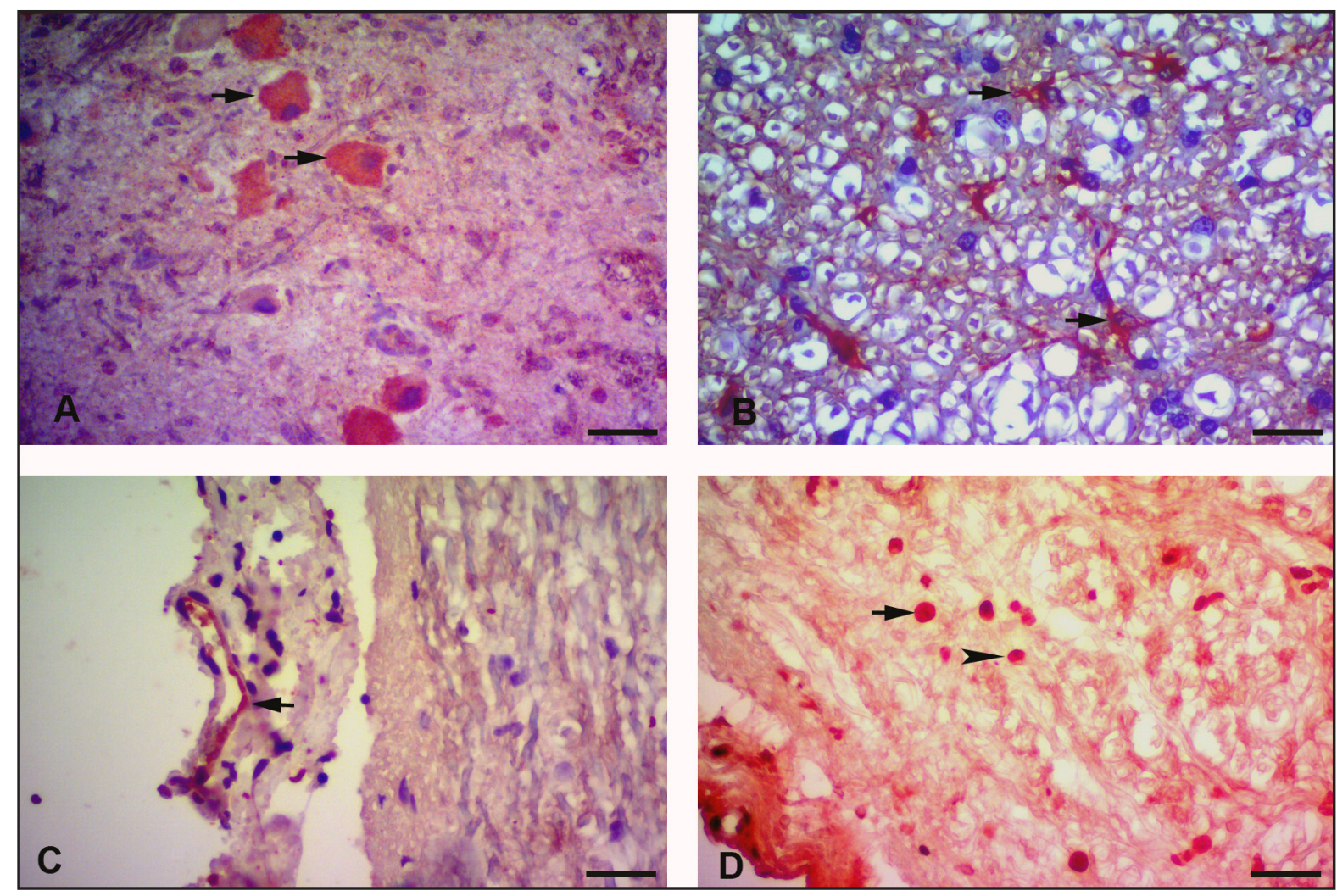

Figure 3. A- iNOS positive neurons. (arrows). IHC [Bar= $25 \mu \mathrm{m}]$. B- iNOS positive glial cells (arrows). IHC [Bar= $25 \mu \mathrm{m}]$. C- eNOS expressions in vessel endothelial cells. (arrow) IHC [Bar= $50 \mu \mathrm{m}]$. D- TUNEL positive neurons (arrow) and glial cells (arrow head). TUNEL [Bar= $25 \mu \mathrm{m}]$.

\section{DISCUSSION}

As a result of the study, it is detected that both eNOS and iNOS expressions take place in control brain tissue but these expressions increase significantly in infected animals' brain tissues with $\operatorname{LM}(P<0.05)$. The relationship between eNOS and iNOS expressions in brains of sheep with listerial meningoencephalitis and apoptosis is examined in the current study. This is the first conducted study in terms of its research subject, which is sheep with listerial meningoencephalitis.

It is observed that iNOS expression in brains of sheep with listeriosis increases in neurons, glial cells and blood vessels [6,20,24]. Moreover, it is found that eNOS expression seriously increases especially in vessel endotheliums, neurons and glial cells in brains of animals with listeriosis. As far as we know, eNOS expression has not been examined in sheep with listeriosis before. However, it is stated that eNOS expression increases as a result of viral diseases of central nervous system or autoimmune encephalomyelitis $[5,8]$.

There are some studies which examine nNOS and iNOS expressions in animals with listeriosis. Although eNOS expression and relationship with apoptosis has not been revealed in these studies, it is notified that nNOS and iNOS expressions increase in neurons and astrocytes as a consequence of bacterial meningoencephalitis. It is also reported that this increase progresses symptoms and lesions of illness and could be effective upon listerial meningoencephalitis pathogenesis [6,20,24].

$\mathrm{NO}$ is effective in regulating various cellular functions [18]. NO can be reversed with various target molecules thanks to its free radical structure and it can participate in redox reactions which may cause irreversible reactions [22]. Chemical reactivity and large quantities of NO during inflammation indicate that NO plays a crucial role in regulating tissue damages which emerge during oxidative stress. On the other hand, its main role is still controversial because NO can both increase and also prevent tissue and cellular damage. Various studies point out that NO has a potential to increase cellular damages $[8,17,20]$. In contrast, NO can serve a protective function during oxidative stress. NO can help reducing oxidative stress by suppressing radical species $[4,23]$. It is reported that NO provides protection against oxidant-related cells and tissues 
$[3,26]$. While some studies stress that NO has potential to induce apoptosis $[1,15]$, some of them state that NO will inhibit this cell death mode $[11,12]$.

It is also specified that increase of iNOS expression within hypothalamus neurons can trigger neuronal apoptosis and neurotoxicity [25]. In addition, it is reported that high iNOS expression level in central nervous system increases glial apoptosis [19]. In another study, it is stated that eNOS and iNOS expressions increase in consequence of viral diseases and thus trigger apoptosis [2]. The current study draws attention to a serious increase in listerial meningoencephalitis eNOS and iNOS expressions [6,20,24] and also in number of neurons and glial cells that undergo apoptosis in comparison with controls $(P<0.05)$. These results obtained from the study are interpreted as similar to results of different diseases, which suggests increase of eNOS and iNOS may trigger apoptosis. However, it is also stated that increase of NO levels can prevent apoptosis on the ground of previous studies $[11,12]$. On the other hand, the opposite situation has been observed in our study. A cell's decision to undergo apoptosis is the result of a change in the balance between the antiapoptotic and proapoptotic forces within the cell. Accumulated data show that relevant Physiological levels of NO contribute to this balance by suppressing apoptotic way at multiple levels with various mechanisms. Higher levels of NO production damage cellular protective mechanisms and lead balance to apoptotic death in some cell types [7].

Consequently, results obtained through the study show that eNOS and iNOS expressions and significantly increase of neuronal and glial cells leading to apoptosis observed in sheep with listeriosis. According to these results, in listeriosis neurons and glial cells may be sources of both eNOS and iNOS. While eNOS and iNOS increase within physiological limits inhibited cellular apoptosis, high levels obtained in our study may have triggered apoptosis. Along with these results, eNOS and iNOS may have a role in tissue and cellular damages and emerging nervous symptoms within listerial meningoencephalitis pathology in sheep. More detailed studies including cytokine in inflammatory response and examining caspase activities should be conducted in order to provide more information about this issue.

\section{CONCLUSIONS}

As a result, eNOS and iNOS expression in neurons and glial cells significantly increased in listeriosis in sheep. With this increase, the number of neurons and glial cells that have undergo apoptosis was found to be higher than the controls. Although more detailed research is needed in this regard, this suggests that NO synthesis may play an important role in the pathology of listerosis in sheep.

\section{MANUFACTURERS \\ ${ }^{1}$ Thermo Fisher Scientific. Waltham, MA, USA. \\ ${ }^{2}$ Novus Biologicals. Centennial, CO, USA. \\ ${ }^{3}$ Roche. Basel, Switzerland.}

Acknowledgements. This study was retrospectively produced from tissue blocks of dead animals diagnosed with Listeriosis and no ethical committee decision is required.

Funding. This study was supported by the Comission of Scientific Research Projects of Cumhuriyet University (Project no: V-076).

Declaration of interest. The authors report no conflicts of interest. The authors alone are responsible for the content and writing of the paper.

\section{REFERENCES}

1 Blanco F.J., Ochs R.L., Schwarz H. \& Lotz M. 1995. Chondrocyte apoptosis induced by nitric oxide. American Journal of Pathology. 146(1): 75-85.

2 Dincel G.C. \& Kul O. 2015. eNOS and iNOS trigger apoptosis in the brains of sheep and goats naturally infected with the border disease virus. Histology and Histopathology. 30: 1233-1242.

3 Gaboury J., Woodman R.C., Granger D.N., Reinhardt P. \& Kubes P. 1993. Nitric oxide prevents leukocyte adherence: role of superoxide. American Journal of Physiology. 265(3): 862-867.

4 Goss S.P., Hogg N. \& Kalyanaraman B. 1995. The antioxidant effect of spermine NONOate in human low-density lipoprotein. Chemical Resourch in Toxicology. 8(5): 800-806.

5 Hooper D.C., Ohnishit S.T., Kean R., Numagamit Y., Dietzschold B. \& Koprowski H. 1995. Medical Sciences Local nitric oxide production in viral and autoimmune diseases of the central nervous system. Proceedings of the National Academy of Sciences of the United States. 92: 5312-5316. 
6 Jungi T.W., Pfister H., Sager H., Fatzer R., Vandevelde M. \& Zurbriggen A. 1997. Comparison of inducible nitric oxide synthase expression in the brain of Listeria monocytogenes-infected cattle, sheep and goats and in macrophages stimulated in vitro. Infect. Immunology. 65: 5279-5288.

7 Kim Y.M., Bombeck C.A. \& Billiar T.R. 1999. Nitric oxide as a bifunctional regulator of apoptosis. Circulation Research. 84: 253-256.

8 Kim S., Moon C., Wie M., Kim H., Tanuma N., Matsumoto Y. \& Shin T. 2000. Enhanced expression of constitutive and inducible forms of nitric oxide synthase in autoimmune encephalomyelitis. Journal of Veterinary Sciences. 1: 11-17.

9 Krueger N., Low C. \& Donachie W. 1995. Phenotypic characterization of the cells of the inflammatory response in ovine encephalitic listeriosis. Journal of Comparative Pathology. 113: 263-275.

10 Leong S.K., Ruan R.S. \& Zhang Z. 2002. A critical assessment of the neurodestructive and neuroprotective effects of nitric oxide. Annals of the New York Academy of Sciences. 962: 161-181.

11 Mannick J.B., Asano K., Izumi K., Kieff E. \& Stamler J.S. 1994. Nitric oxide produced by human B lymphocytes inhibits apoptosis and Epstein-Barr virus reactivation. Cell. 79(7): 1137-1146.

12 Matthys P., Froyen G., Verdot L., Huang S., Sobis H., Van Damme J., Vray B., Aguet M. \& Billiau A. 1995. IFNgamma receptor-deficient mice are hypersensitive to the anti-CD3-induced cytokine release syndrome and thymocyte apoptosis. Protective role of endogenous nitric oxide. Journal of Immunology. 155(8): 3823-3829.

13 Maxie M.G. \& Youssef S. 2007. Nervous system. In: Maxie M.G. (Ed). Jubb K. \& Palmer's Pathology of domestic animals. Philadelphia: Saunders-Elsevier, pp.405- 408

14 Messmer U.K., Ankarcrona M., Nicotera P. \& Brune B. 1994. p53 Expression in nitric oxide-induced apoptosis. FEBS Letters. 355: 23-26.

15 Messmer U.K. \& Reed U.K. 1996. Brüne B. Bcl-2 protects macrophages from nitric oxide-induced apoptosis. Journal of Biological Chemistry. 271(33): 20192-20197.

16 Moncada S., Palmer R.M.J. \& Higgs E.A. 1991. Nitric oxide: physiology, pathophysiology, and pharmacology. Pharmacological Reviews. 43(2): 109-142.

17 Mulligan M.S., Warren J.S., Smith C.W., Anderson D.C., Yeh C.G., Rudolph A.R. \& Ward P.A. 1992. Lung injury after deposition of IgA immune complexes. Requirements for CD18 and L-arginine. Journal of Immunology. 148(10): 3086-3092.

18 Nathan C. 1992. Nitric oxide as a secretory product of mammalian cells. The FASEB Journal. 6(12): 3051-3064.

19 Pender M.P. \& Rist J.M. 2001. Apoptosis of inflammatory cells in immune control of the nervous system: Role of glia. Glia 36: 137-144.

20 Pfister H., Remer K.A., Brcic M.,. Fatzer R., Christen S., Leib S. \& Jungi T.W. 2002. Inducible Nitric Oxide Synthase and Nitrotyrosine in Listeric Meningoencephalitis: A Cross-species Study in Ruminants. Veterinary Pathology. 39: 190-199.

21 Radi R., Beckman J.S., Bush K.M. \& Freeman B.A. 1991. Peroxynitrite-induced membrane lipid peroxidation: the cytotoxic potential of superoxide and nitric oxide. Archives of Biochemistry and Biophysics. 88(2): 481-487.

22 Rubbo H., Darley-Usmar V. \& Freeman B.A. 1996. Nitric oxide regulation of tissue free radical injury. Chemical Research in Toxicology. 9(5): 809-820.

23 Rubbo H., Radi R., Trujillo M., Telleri R., Kalyanaraman B., Barnes S., Kirk M. \& Freeman B.A. 1994. Nitric oxide regulation of superoxide and peroxynitrite-dependent lipid peroxidation. Formation of novel nitrogen-containing oxidized lipid derivatives. Journal of Biological Chemistry. 69(42): 26066-26075

24 Shin T., Weinstock D., Castro M.D., Acland H., Walter M., Kim H.Y. \& Purchase H.G. 2000. Immunohistochemical Study of Constitutive Neuronal and Inducible Nitric Oxide Synthase in the Central Nervous System of Goat with Natural Listeriosis. Journal of Veterinary Science. 1(2): 77-80

25 Wang C., Hikim A.S., Ferrini M., Bonavera J.J., Vernet D., Leung A., Lue Y.H., Gonzalez-Cadavid N.F. \& Swerdloff R.S. 2002. Male reproductive ageing: using the brown Norway rat as a model for man. Novartis Foundation symposium 242: 82-95.

26 Wink D.A., Hanbauer I., Krishna M.C., DeGraff W., Gamson J. \& Mitchell J.B. 1993. Nitric oxide protects against cellular damage and cytotoxicity from reactive oxygen species. Proceedings of the National Academy of Sciences of the United States of America. 90(21): 9813-9817. 
M.Ö. Karayiğit. 2018. Nitric Oxide Synthase Expression in Naturally Infected Sheep Brain with Listeria monocytogenes and Relationship with Cell Death. Acta Scientiae Veterinariae. 46: 1617.

27 Xie Q.,W., Cho H.J., Calaycay J., Mumford R.A., Swiderek K.M., Lee T.D., Diang A., Troso T. \& Nathan C. 1992. Cloning and characterization of inducible nitric oxide synthase from mouse macrophages. Science. 256(5051): 225-228.

28 Zachary J.F. 2012. Listeriosis. In: Zachary J.F. \& McGavin M.D. (Eds). Pathologic Basis of Veterinary Disease. St. Louis: Elsevier-Mosby, pp.192-195. 